\title{
Internal Leakage Diagnosis of a Hydraulic Cylinder Based on Optimization DBN Using the CEEMDAN Technique
}

\author{
Peng Zhang $\mathbb{D}^{1,2}$ and Xinyuan Chen $\mathbb{D i D}^{1,2}$ \\ ${ }^{1}$ Key Laboratory of Metallurgical Equipment and Control Technology (Wuhan University of Science and Technology), \\ Ministry of Education, Wuhan, China \\ ${ }^{2}$ Hubei Key Laboratory of Mechanical Transmission and Manufacturing Engineering, \\ Wuhan University of Science and Technology, Wuhan 430081, China \\ Correspondence should be addressed to Xinyuan Chen; chenxinyuan@wust.edu.cn
}

Received 19 September 2020; Revised 30 January 2021; Accepted 6 March 2021; Published 13 March 2021

Academic Editor: Li Qing

Copyright (c) 2021 Peng Zhang and Xinyuan Chen. This is an open access article distributed under the Creative Commons Attribution License, which permits unrestricted use, distribution, and reproduction in any medium, provided the original work is properly cited.

\begin{abstract}
Internal leakage diagnosis in a hydraulic cylinder is a key technique for the maintenance of hydraulic systems. However, it is difficult to diagnose the internal leakage under different low loads. To solve this problem, a novel fault diagnosis method based on the optimization deep belief network (DBN) combined with the complete ensemble empirical mode decomposition with adaptive noise (CEEMDAN) technique is proposed to treat the collected AE signals. The raw AE signals are decomposed into a set of intrinsic mode functions (IMFs) by using CEEMDAN. Subsequently, according to the decreasing order of the Pearson correlation coefficient values, the first five IMFs are selected for signal reconstruction to suppress the abnormal interference from noise. The reconstructed signals are regarded as the input of the optimization DBN, and the particle swarm optimization simulated annealing (PSOSA) algorithm is adopted to identify the four internal leakage levels. The experimental results show that the proposed method exhibits a higher classification accuracy than other methods under different low loads. This result validates the effectiveness and superiority of the proposed approach to realize internal leakage diagnoses under different low loads.
\end{abstract}

\section{Introduction}

Hydraulic systems are widely applied in industry as actuators in hydraulic systems [1-3]. Internal leakage is a major failure mode caused by the wear of the piston seal that closes the gap between the movable piston and cylinder wall [4]. The detection of internal leakage is critical in practical applications because such leakage can affect the dynamic performance of the system. However, internal leakage cannot be detected until the actuator seal is completely damaged, and the actuator fails to respond to a control signal. Moreover, the detection of internal leakage is challenging due to the associated concealment. In addition, online nonintrusive diagnosis of internal leakage cannot be easily realized because of the dynamic working conditions of hydraulic cylinders. Hence, this paper proposes a method to realize automatic internal leakage diagnoses under different loads.
The existing methods for the fault diagnoses of internal leakage in hydraulic cylinders can be categorized into two types, namely, model-based and data-driven methods. Among model-based methods, a linear model-based approach with an adaptive threshold [5] and extended Kalman filter- (EKF-) based fault detection scheme [6] have been developed to detect different types of leakage in hydraulic actuators. However, due to the uncertainty and nonlinearity in hydraulic systems, it is difficult to establish a precise model. Consequently, researchers have adopted data-driven methods to realize fault diagnoses. Data-driven methods usually include two main steps: fault feature extraction and selection using signal processing techniques, and fault classification using classifiers. Many features based on pressure signals have been found to be sensitive to internal leakage, such as the root mean square (RMS) values of level two detailed coefficients obtained using the wavelet 
transform [7-9], five features extracted through wavelet packet analysis [10], and the instantaneous amplitude of the first IMF obtained through the EMD technique [11]. However, to implement the abovementioned schemes, a baseline value must be predetermined, and this value cannot be automatically adjusted with the change in the working conditions. Hence, $\mathrm{Li}$ et al. proposed a featured temporal segmentation method and an AdaBoost-BP detector to automatically evaluate the internal leakage faults in hydraulic actuators [4]. Nevertheless, such traditional datadriven methods exhibit several notable deficiencies: (1) the features input to the classifiers are designed by diagnosticians depending on prior knowledge regarding the signal processing techniques and diagnostic expertise. (2) The adopted classifiers have shallow architectures, which limit the capacity of such classifiers to learn the complex nonlinear relationships in fault diagnosis scenarios [12]. These limitations can be overcome by using deep learning (DL) algorithms. The key property of deep learning models is the multiple layer structure. Using the multiple hidden layers stacked hierarchically, a deep learning model can realize the highly complicated transformation and abstraction of raw signals $[13,14]$. Guo et al. adopted a convolutional neural network (CNN) that employed raw strain signals to output the internal leakage of the hydraulic cylinder [15]. However, the strain caused by microflows is extremely small under low pressure, that is, for pressure values lower than $7 \mathrm{MPa}$, and the abovementioned method cannot effectively diagnose the internal leakage in such cases. In other words, the existing methods cannot effectively diagnose internal leakage under different low loads.

In contrast to the internal leakage detection technologies based on the dynamic pressure or strain, the acoustic emission (AE) technology, as a nondestructive testing (NDT) technique with an extremely high sensitivity, can be used to detect weak internal leakage signals without hindering operations [16-20]. Furthermore, the AE technology exhibits several advantages over other nondestructive testing (NDT) techniques such as the vibration technology [21]. First, the defects are easier to detect because of the enhanced signal to noise ratio. Second, because this approach can detect mechanical abnormalities at high frequencies, it is highly sensitive compared to vibration techniques. In addition, the $\mathrm{AE}$ technology can be applied to all types of machinery, regardless of speed [22]. Nevertheless, owing to the use of a highly sensitive transducer, the measured raw signal, which often includes noise and irrelevant and redundant signals, must be subjected to noise removal to ensure reliable fault diagnosis. CEEMDAN-based denoising methods can self-adaptively decompose a signal into several monocomponents according to the local characteristic time scales of the signal, which can ensure a high decomposition performance in nonlinear and nonstationary processes [23]. Moreover, under different working loads of the hydraulic cylinder, the difference in the raw leakage signals of identical patterns increases, which hinders the fault diagnosis process. In this case, Pearson correlation coefficients can be used to account for the main characteristics that differentiate the fault modes while eliminating the difference caused by the different working conditions $[24,25]$. Therefore, in this study, a reconstruction denoising strategy combining CEEMDAN with Pearson correlation coefficients is established to obtain the denoised signal. First, the sampled AE signals are decomposed into different IMFs by using CEEMDAN. Subsequently, the Pearson correlation coefficients between the raw signals and each IMF are calculated. The reconstructed data based on the IMFs with large Pearson correlation coefficients are obtained for further analysis.

The deep belief network (DBN) is a promising tool to realize fault characteristic mining and intelligent diagnosis in the case of massive data $[19,26-28]$. In contrast to the $\mathrm{CNN}$, the DBN avoids the process of transforming onedimensional time series data. Moreover, the DBN does not rely on manual feature extraction and selection, and thus, it can automatically learn effective information from the raw signals. To reduce the influence of manual parameter setting on fault diagnosis accuracy, the hybrid particle swarm optimization simulated annealing (PSOSA) algorithm [29, 30] is adopted to identify the optimal structure of the trained DBN [31]. Moreover, experiments are conducted using a gas-hydraulic pressurized cylinder, which is easy to disassemble and can operate under low load conditions.

Overall, this paper proposes a method involving the optimization DBN in combination with the CEEMDAN technique. First, the CEEMDAN combined with the Pearson correlation coefficients is employed to eliminate noise from the raw AE signals for four different internal leakage levels. Subsequently, the denoised signals are directly fed to the optimization DBN by the PSOSA to identify the levels of internal leakage.

\section{Theoretical Framework}

2.1. CEEMDAN Theory. The process flow of the CEEMDAN algorithm can be summarized as follows [32, 33]:

Step 1: Add a white noise series to the investigated signal, that is, $x^{(i)}=x+\beta w^{(i)}$, where $i=1,2,3, \ldots, I$. Decompose each $x^{(i)}$ by using the EMD to obtain the corresponding $d_{1}^{(i)}$. Next, calculate the first CEEMDAN mode $\overline{d_{1}}=(1 / I) \sum_{i=1}^{I} d_{1}^{(i)}$ and first residue $r_{1}=x-\overline{d_{1}}$. Step 2: Decompose each $r_{1}+\varepsilon_{1} E_{1}\left(w^{(i)}\right)$ by using the EMD, where $i=1,2,3, \ldots, I, E_{k}$ represents the $k$-th mode component of the given signal obtained through the EMD calculation. Define the second CEEMDAN mode as $\overline{d_{2}}=(1 / I) \sum_{i=1}^{I} E_{1}\left(r_{1}+\varepsilon_{1} E_{1}\left(w^{(i)}\right)\right)$. For $k=2$, $\ldots, K$, calculate the kth residue, $r_{k}=r_{k-1}-\overline{d_{k}}$;

Step 3: Decompose each $r_{k}+\varepsilon_{k} E_{k}\left(w^{(i)}\right)$ by using the EMD, where $i=1, \ldots, I$. Calculate the $k+1$ CEEMDAN mode as $\overline{d_{k+1}}=(1 / I) \sum_{i=1}^{I} E_{1}\left(r_{k}+\varepsilon_{k}\left(w^{(i)}\right)\right)$.

2.2. DBN Principle. The DBN consists of one visible layer and three hidden layers, as shown in Figure 1. The learning process of DBN includes two stages: the unsupervised pretraining stage and the supervised fine-tuning stage. In the supervised fine-tuning stage, the labelled target data are input to the model, and the parameters are updated [34, 35]. 


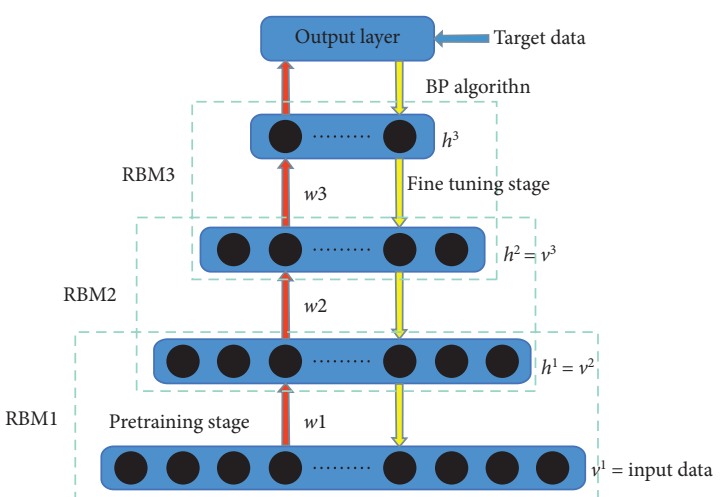

Figure 1: Architecture of the DBN.

Suppose that there exist I visible units and $J$ hidden units in an RBM. The states of the visible layer and hidden layer are represented as $v$ and $h$, respectively. The RBM energy function can be defined as follows:

$$
E(v, h \mid \theta)=-\sum_{i=1}^{I} a_{i} v_{i}-\sum_{j=1}^{J} b_{j} h_{j}-\sum_{i=1}^{I} \sum_{j=1}^{J} w_{i j} v_{i} h_{j},
$$

where $\theta=\left\{w_{i j}, b_{j}, a_{i}\right\} ; \theta$ represents the model parameters; $v_{i}$ is the visible unit $i ; h_{j}$ is the hidden unit $j ; w_{i j}$ is the weight connecting the visible unit $i$ and hidden unit $j ; a_{i}$ is the offset of the visible unit $i$; and $b_{j}$ is the offset of the hidden unit $j$.

The contrastive divergence (CD) algorithm is adopted to update the model parameters as follows:

$$
\begin{aligned}
\Delta w & =\varepsilon_{w}\left(\left\langle v h^{T}\right\rangle_{\text {data }}-\left\langle v h^{T}\right\rangle_{\text {recon }}\right), \\
\Delta a & =\varepsilon_{a}\left(\langle v\rangle_{\text {data }}-\langle v\rangle_{\text {recon }}\right), \\
\Delta b & =\varepsilon_{a}\left(\langle v\rangle_{\text {data }}-\langle v\rangle_{\text {recon }}\right), \\
w & \leftarrow w+m \Delta w, \\
a & \leftarrow a+m \Delta a, \\
b & \leftarrow b+m \Delta b,
\end{aligned}
$$

where $\varepsilon_{w}, \varepsilon_{a}, \varepsilon_{b}$ denote the learning rates of the weights between 0 and $1 ; a$ and $b$ represent the biases of the hidden layer and visible layer, respectively; $\langle\cdot\rangle_{\text {data }}$ and $\langle\cdot\rangle_{\text {recon }}$ refer to the expectation derived using the training data distribution and reconstructed data, respectively.

2.3. Hybrid PSO-SA Optimization Method. The following steps are involved in the hybrid PSOSA algorithm [36-38]:

Step 1: Randomly initialize the parameters, including the position $x_{i}^{0}$, velocity $v_{i}^{0}$, and iterations. Calculate the fitness value of each particle $F\left(x_{i}\right)$ by using the fitness function defined in

$$
F\left(x_{i}\right)=\frac{n_{\text {error }}}{N},
$$

where $n_{\text {error }}$ refers to the number of misclassified data, and $N$ refers to the total number of data. Next, update the best position of a particle $x_{i p \text { best }}^{k}$ and the best particle position of the swarm $x_{g \text { best }}^{k}$ according to the history. Initialize temperature $T=\left(-F\left(x_{\text {gbest }}^{k}\right) / \ln (0.2)\right)$.

Step 2: Update the positions and velocities of all the particles by using the following function:

$$
\begin{aligned}
v_{i}^{k+1} & =w v_{i}^{k}+c_{1} r_{1}\left(x_{i p \text { best }}^{k}-x_{i}^{k}\right)+c_{2} r_{2}\left(x_{g \text { best }}^{k}-x_{i}^{k}\right), \\
x_{i}^{k+1} & =x_{i}^{k}+v_{i}^{k+1}, \\
w & =w_{\max }-\frac{\left(w_{\max }-w_{\min }\right) k}{T}, \\
c_{1} & =c_{\max }-\frac{\left(c_{\max }-c_{\min }\right) k}{T}, \\
c_{2} & =c_{\max }+\frac{\left(c_{\max }-c_{\min }\right) k}{T},
\end{aligned}
$$

where $w$ is the inertia coefficient, $w \in[0,1] . c_{1}$ and $c_{2}$ are cognitive parameters. $r_{1}$ and $r_{2}$ are random values in the interval $[0,1]$.

Step 3: Evaluate whether the new solution is accepted by using the metropolis acceptance criteria as follows:

$$
\Delta f=F\left(x_{i}^{k+1}\right)-F\left(x_{i}^{k}\right) ; \quad \text { if } \Delta f<0 ; x_{i}^{k}=x_{i}^{k+1} .
$$

Otherwise, calculate the acceptance probability $p=\exp (-\Delta f / T)$.

$$
\text { if } p>\operatorname{rand}(0,1), x_{i}^{k}=x_{i}^{k+1} ; \quad \text { else } x_{i}^{k}=x_{i}^{k} .
$$

Step 4: Update the best position of a particle $x_{i p b e s t}^{k}$ and the best particle position of the swarm $x_{\text {gbest }}^{k}$ considering the history.

Step 5: If $F\left(x_{\text {gbest }}^{k}\right) \leq 0.01$, terminate the cycle, and output the best solution and objective. Otherwise, gradually decrease the temperature $T=T * 0.95$, and return to step 3 .

\section{Experimental Setup}

3.1. Test Rig. As shown in Figure 2(a), the test rig consists of a gas-hydraulic pressurized cylinder 1 , Jack 2 , and pneumatic device 3.

The operating principle of the gas-hydraulic pressurized cylinder is shown in Figure 3. The red, green, blue, purple, and black regions correspond to the high-pressure air, lowpressure air, pressure-free hydraulic oil, preloading hydraulic oil, and high-pressure hydraulic oil, respectively. AE sensor 3 with an electromagnet is fixed on the mounting plane. The piston of rod 2 uses a y-sealing ring. Notches with different sizes are set on the sealing rings for a load of $1 \mathrm{MPa}$ and $2 \mathrm{MPa}$ to simulate four internal leakage levels corresponding to no internal leakage, small internal leakage, medium internal leakage, and large internal leakage. The seal rings with different size notches under $1 \mathrm{MPa}$ are illustrated in Figure 2(b). The leaking oil flows out through the P1 port, and the internal leakage rate of the flow is determined using 

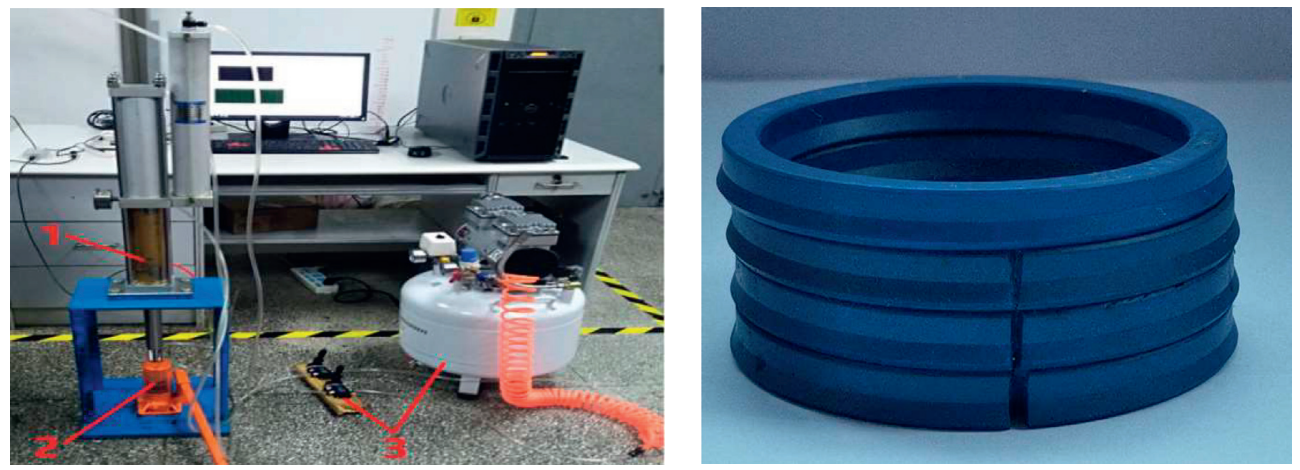

Figure 2: (a) Image of the test rig. (b) Image of the seal rings with notches of different sizes.

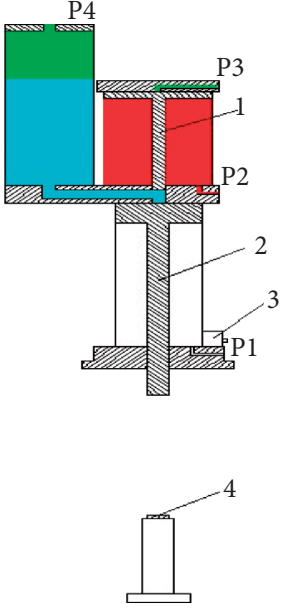

(a)

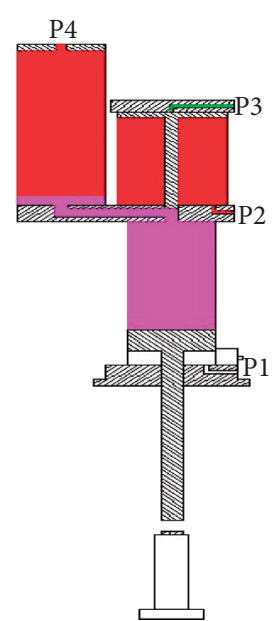

(b)

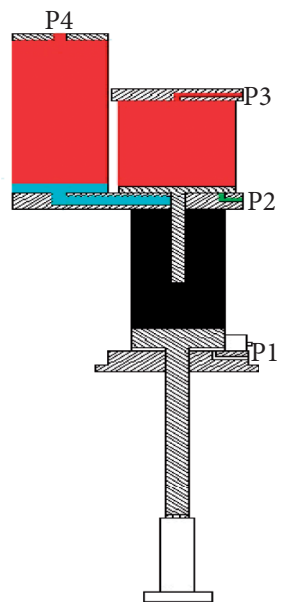

(c)

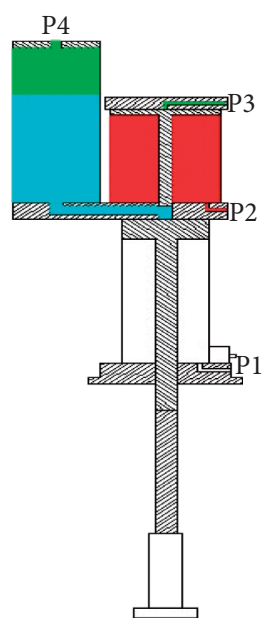

(d)

Figure 3: Schematic of the gas-hydraulic pressurized cylinder.

a measuring cup and stopwatch. The operating principle of the gas-hydraulic pressurized cylinder can be defined as follows:

(1) As shown in Figure 3(a), the P2 port connects the high-pressure air, the $\mathrm{P} 3$ and $\mathrm{P} 4$ ports connect the low-pressure air, the $\mathrm{P} 1$ port is the drain port, rods 1 and 2 retract, and the hydraulic oil is located in the oil tank.

(2) As shown in Figure 3(b), the P4 port connects the high-pressure air and preloading hydraulic oil. The hydraulic oil enters into nonrod chamber of the cylinder, and rod 2 extends.

(3) As shown in Figure 3(c), the P3 port connects the high-pressure air, and P2 port connects the lowpressure air. Rod 1 extends, thereby pressurizing the hydraulic oil. The leaking hydraulic oil flows out through the P1 port.

(4) As shown in Figure 3(d), the P2 port connects the high-pressure air, $\mathrm{P} 3$ port connects the low-pressure air, rod 1 retracts, and pressure relief occurs. Rod 2 is compressed by Jack 4, and the hydraulic oil flows back to the oil tank.
3.2. AE Sensor and Data Acquisition Device. As shown in Figure 4(a), AE sensor 1 is the PXR04 device manufactured by Changsha Pengxiang Electronic Technology Co. LTD. This device has a frequency range and resonant frequency of $30-140 \mathrm{kHz}$ and $40 \mathrm{kHz}$, respectively. Amplifier 2 shown in Figure 4(b) has a fixed gain of $100 \mathrm{~dB}$. Data acquisition card 3 pertains to NI-6340 manufactured by National Instruments; this device has a maximum acquisition frequency and output voltage amplitude of $400 \mathrm{kHz}$ and $10 \mathrm{~V}$, respectively.

\section{Internal Leakage Diagnosis Method Based on the Optimization DBN}

The proposed diagnosis method based on the optimization DBN can be described as follows:

Step 1: Define four internal leakage levels of the hydraulic cylinder under two loads (1 and $2 \mathrm{MPa}$ ). Collect raw $\mathrm{AE}$ signals and decompose the signals into a set of IMFs through the CEEMDAN.

Step 2: Calculate the Pearson correlation coefficient between each IMF and the raw AE signals. Next, select the first five sensitive IMFs to obtain the reconstructed 

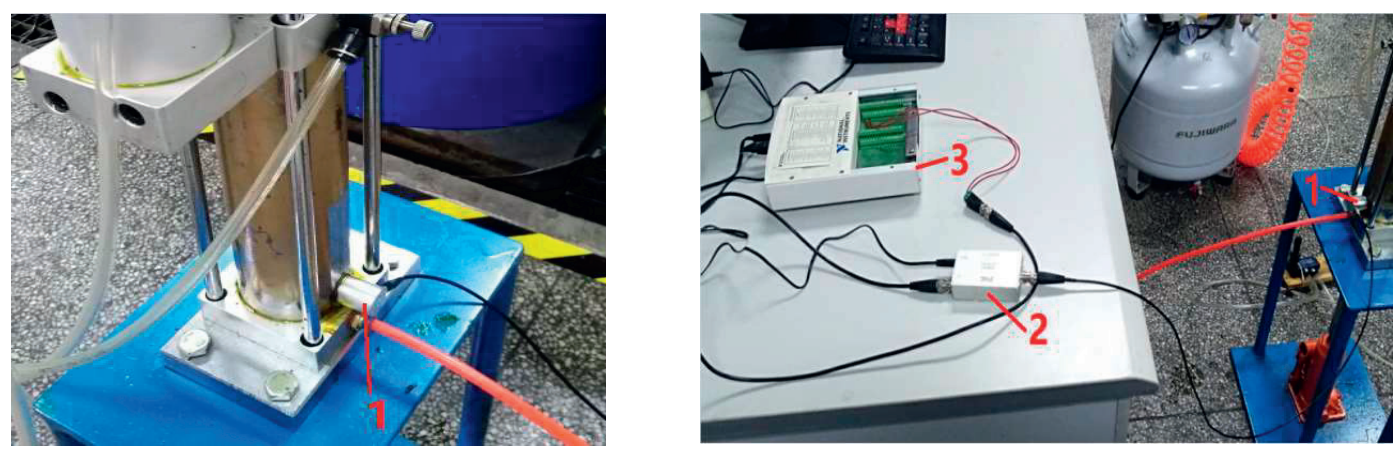

Figure 4: (a) Image of the AE sensor. (b) Image of the data acquisition device.

signals by considering the decreasing correlation coefficient values.

Step 3: Divide the reconstructed signals into training and testing samples. Train the optimization DBN by using the PSOSA algorithm and training samples. Use this network to classify the internal leakage levels by considering the testing samples. These steps are implemented to apply the proposed method to diagnose four internal leakage levels, and the results are recorded.

4.1. Signal Reconstruction and Data Description. According to the Shannon sampling theorem, the sampling frequency of the data acquisition card is set as $400 \mathrm{kHz}$. The AE signals in different internal leakage levels under a load of 1 and $2 \mathrm{MPa}$ are shown in Figures 5 and 6, respectively.

The original signals are preprocessed using CEEMDAN. The Pearson correlation coefficient represents the correlation between each IMF and the original signal. The Pearson correlation coefficient between $\operatorname{IMF}_{k}(t)$ and the original signal data sequence $S(t)$ is calculated as follows [24]:

$$
R_{k}=\frac{\sum_{t=1}^{T}\left(\mathrm{IMF}_{k}(t)-\overline{\mathrm{IMF}_{k}(t)}\right)(S(t)-\overline{S(t)})}{\sqrt{\sum_{t=1}^{T}\left(\mathrm{IMF}_{k}(t)-\overline{\mathrm{IMF}_{k}(t)}\right)^{2}(S(t)-\overline{S(t)})^{2}}}
$$

where $T$ is the number of points in the original signal data sequence; $\overline{S(t)}$ and $\overline{\mathrm{IMF}_{k}(t)}$ denote the mean values of $S(t)$ and $\operatorname{IMF}_{k}(t)$, respectively.

To eliminate the noise and enhance the signal to noise ratio, the first five IMFs are used for signal reconstruction. The reconstruction process can be expressed as

$$
\widehat{S}(t)=\mathrm{IMF}_{a}+\mathrm{IMF}_{b}+\mathrm{IMF}_{c}+\mathrm{IMF}_{d}+\mathrm{IMF}_{e},
$$

where $\widehat{S}(t)$ denotes the reconstructed signal; $\mathrm{IMF}_{a}, \mathrm{IMF}_{b}$, $\mathrm{IMF}_{c}, \mathrm{IMF}_{d}$, and $\mathrm{IMF}_{e}$ are the first five IMFs.

In this case study, datasets A and B are established to verify the performance of the proposed method. Datasets A and $\mathrm{B}$ include the data for the four conditions under loads of 1 and $2 \mathrm{MPa}$, respectively. Both datasets contain 100 training samples (each sample has 2000 data points) and 50 testing samples for each condition. The sample distributions for the four conditions in datasets A and B are presented in Table 1.
4.2. Comparison Approach. In this case study, PSOSA is applied to develop the optimization DBN based on the training samples. The parameter settings for the hybrid PSOSA are presented in Table 2. Table 3 lists the values of the parameters for the optimization DBN derived using the PSOSA. The optimal architecture of the DBN is 2000-860284-78-4. The input and output layers consist of 2000 and 4 neurons, respectively, which are set based on the dimensions of the input data and fault patterns. The learning rate and momentum obtained using the PSOSA are 0.48 and 0.23 , respectively.

To illustrate the effectiveness of the proposed method in actual applications of the fault diagnosis for the internal leakage, comparative experiments are conducted considering the following six methods:

(1) DBN method: The original AE signals are used directly as the input of the optimization DBN.

(2) EEMD + DBN: The reconstructed AE signals based on the EEMD method are used as the input of the optimization DBN.

(3) CEEMDAN + DBN: The reconstructed AE signals based on the CEEMDAN method are used as the input of the optimization DBN.

(4) CEEMDAN + BP: The reconstructed AE signals based on the CEEMDAN are used as the input of the BP network. To enable a fair comparison, the BP network and optimization DBN used the same data, architecture, learning rate, momentum, batch size, and epochs.

(5) $T+\mathrm{BP}$ : the original AE signals are preprocessed to extract the time domain features including the mean value, root mean square, peak, skewness, kurtosis value, crest factor, clearness factor, shape factor, and impulse factor. Next, the 9 selected features are used as the input of the BP network with 1 hidden layer. The architecture of the BPNN is 9-78-4.

(6) $T+F+\mathrm{BP}$ : The original AE signals are preprocessed to extract the time and frequency domain features. The 9 time domain features are the same as those for method 5. The 4 frequency domain features include the mean frequency, frequency centre, root mean frequency, and standard deviation frequency. Next, the 13 selected features are used as the input of the 

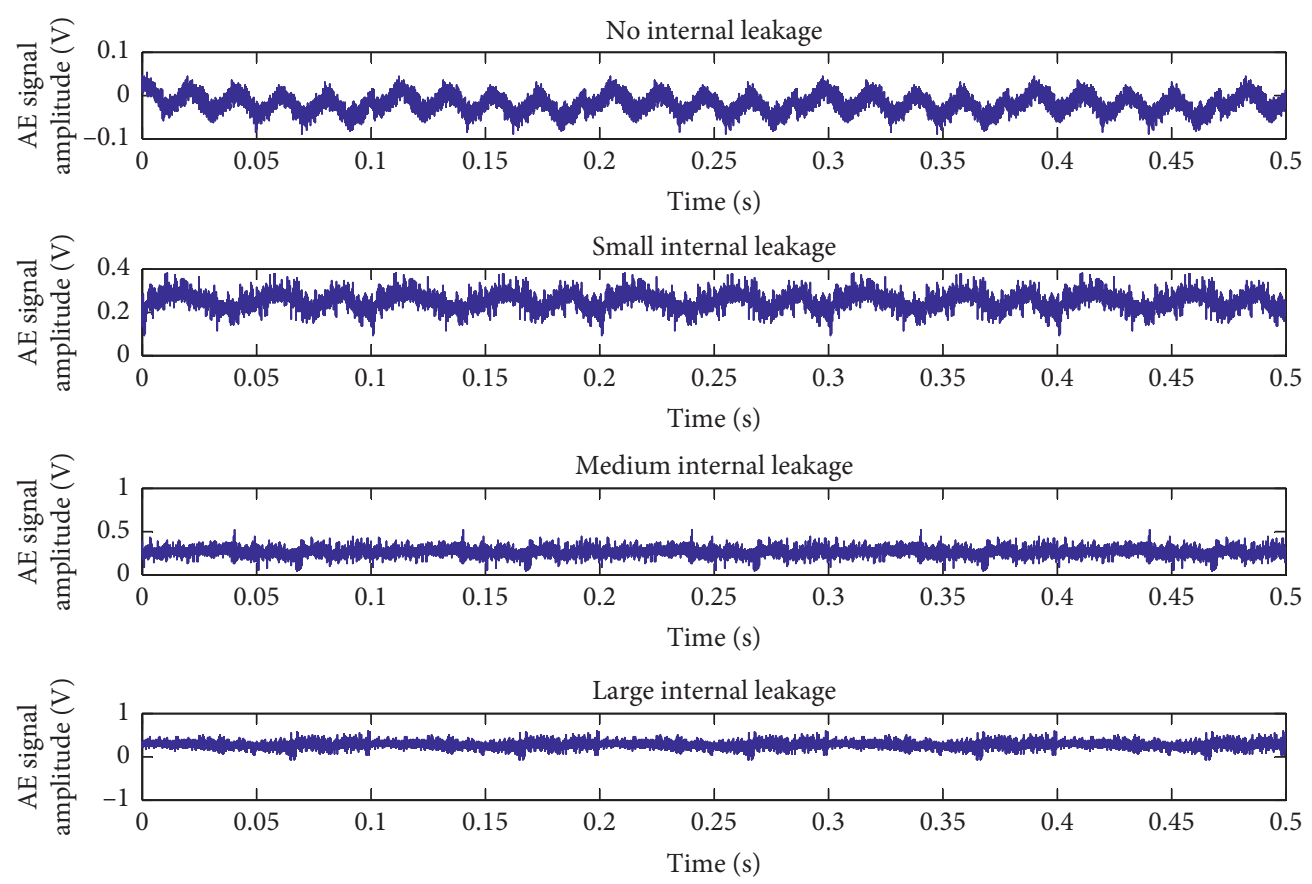

Figure 5: AE signals under a load of $1 \mathrm{MPa}$ in four different internal leakage states.
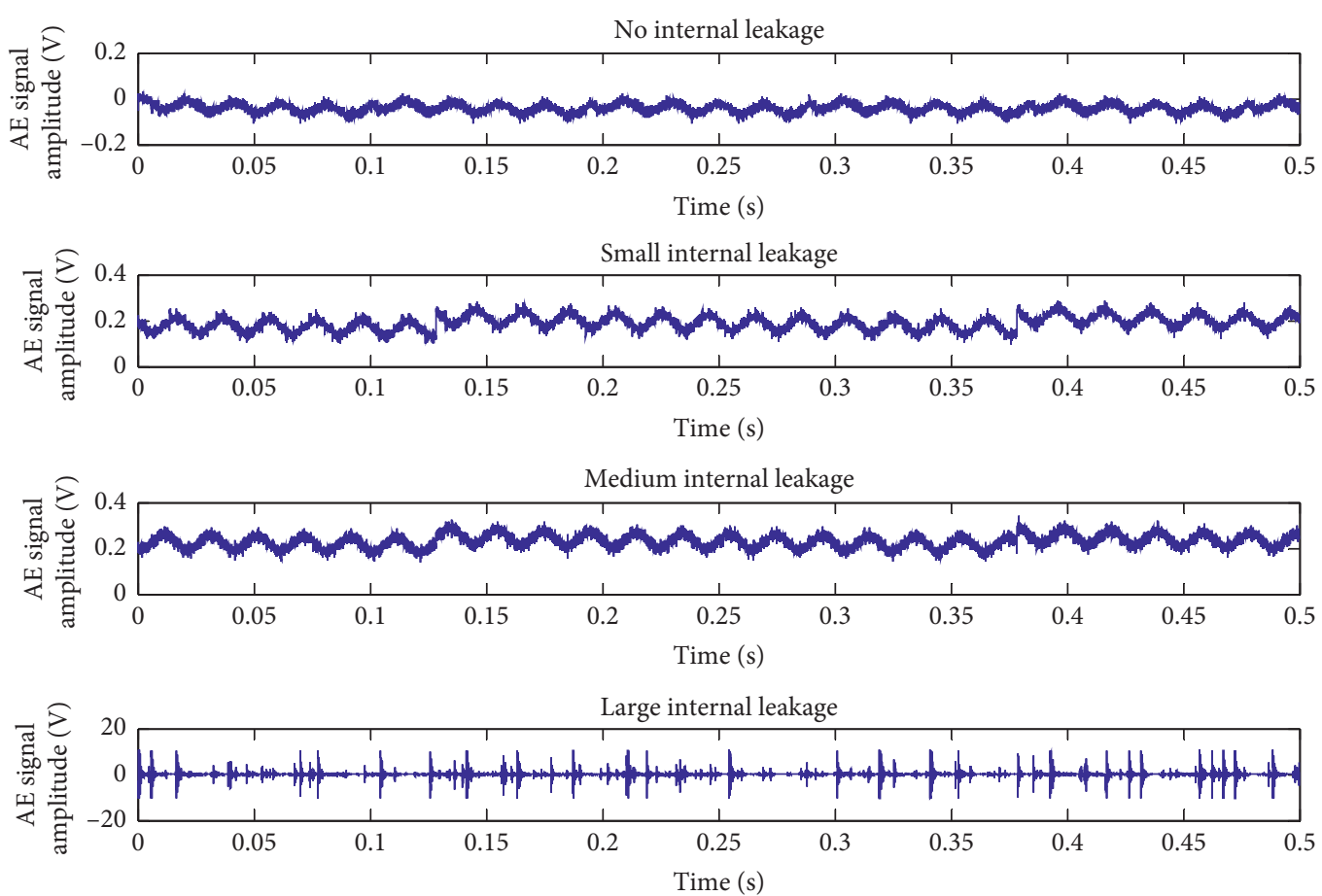

Figure 6: AE signals under a load of $2 \mathrm{MPa}$ in four different internal leakage states.

TABLE 1: Sample distributions under four different conditions.

\begin{tabular}{|c|c|c|c|c|}
\hline Datasets & Load $(\mathrm{MPa})$ & Number of training samples/testing samples & Fault type & Label \\
\hline \multirow{4}{*}{ A } & \multirow{4}{*}{1} & $100 / 50$ & No internal leakage & 1 \\
\hline & & $100 / 50$ & Small internal leakage & 2 \\
\hline & & $100 / 50$ & Medium internal leakage & 3 \\
\hline & & $100 / 50$ & Large internal leakage & 4 \\
\hline \multirow{4}{*}{ B } & \multirow{4}{*}{2} & $100 / 50$ & No internal leakage & 1 \\
\hline & & $100 / 50$ & Small internal leakage & 2 \\
\hline & & $100 / 50$ & Medium internal leakage & 3 \\
\hline & & $100 / 50$ & Large internal leakage & 4 \\
\hline
\end{tabular}


TABLE 2: Parameter settings for the hybrid PSO-SA.

\begin{tabular}{lc}
\hline Parameters & Value \\
\hline Population size of PSO & 20 \\
Number of iterations of PSO & 50 \\
Initial temperature $T$ & 100 \\
Annealing factor & 0.95 \\
Max/min position of a particle & $500 / 10$ \\
Max/min speed of a particle & $1 /-1$ \\
Max/min weight of PSO & $1.5 / 0.5$ \\
Max/min acceleration factor of PSO & $1.5 / 0.5$ \\
\hline
\end{tabular}

TABle 3: Optimization parameters of the DBN.

\begin{tabular}{lc}
\hline Parameters & Value \\
\hline Number of nodes in the input layer & 2000 \\
Number of nodes in the output layer & 4 \\
Number of RBMs & 3 \\
Number of iterations for each RBM & 200 \\
Number of nodes in the first hidden layer (obtained using the PSO-SA) & 860 \\
Number of nodes in the second hidden layer (obtained using the PSO-SA) & 284 \\
Number of nodes in the third hidden layer (obtained using the PSO-SA) & 78 \\
Learning rate of the deep belief network (obtained using the PSO-SA) & 0.48 \\
Momentum of the deep belief network (obtained using the PSO-SA) & 0.23 \\
\hline
\end{tabular}

BP network with 1 hidden layer. The architecture of the BPNN is $13-78-4$.

The six methods are run 20 times, and the average classification accuracy is determined, as listed in Table 4.

The results show that the proposed method exhibits a considerably higher classification accuracy than that of the traditional methods based on the BP network. The BP network has the same architecture as the DBN, and the same reconstructed $\mathrm{AE}$ signals without any feature extraction are used to train the DBN and BP network in methods 3 and 4 . The comparison of the diagnosis accuracy of methods 3 and 4 indicates that the accuracy of the DBN is considerably higher than that of the BP network when managing massive data. This phenomenon occurs because deep learning methods can learn the valuable information from the input data through multiple feature transformations. According to the comparison of the diagnosis accuracy of methods 5 and 6 , different features yield different results, which indicates that the realization of the accurate classification of BP network requires manual intervention to select the proper features to implement the fault diagnosis.

In addition, the DBN combined with the CEEMDAN to realize the denoising in method 3 exhibits a higher accuracy than that of the DBN based on the original AE signals (method 1). This finding demonstrates that the proposed method can effectively denoise signals and enhance the robustness of the fault diagnosis results. Furthermore, the $\mathrm{CEEMD}+\mathrm{DBN}$ outperforms the EEMD $+\mathrm{DBN}$, and in contrast to the EEMD, the CEEMDAN can decrease the reconstruction error and avoid the problem of different numbers of modes for different realizations of the signal plus noise. Furthermore, the proposed method can achieve a higher classification accuracy than that of the other methods in datasets $\mathrm{A}$ and $\mathrm{B}$. The results demonstrate that the proposed method can effectively diagnose the fault types even under load fluctuations.

\section{Results and Discussion}

The Pearson correlation coefficients are used to select the sensitive IMFs for further processing. However, no prior knowledge is available to set the number of sensitive IMFs for further reconstruction. Therefore, an investigation is conducted to determine the number of sensitive IMFs. According to the decreasing correlation coefficient values, the number of sensitive IMFs is increased sequentially from 1 to 12 . The procedures are repeated 20 times, and the average accuracy for the testing samples of datasets A and B is separately determined.

The average classification accuracy varies with the different numbers of selected IMFs, as shown in Figure 7. Initially, the accuracy increases with the number of selected IMFs. However, the accuracy decreases as the number of selected IMFs increases to 12 . The result indicates that, in the initial stage, as the number increases from 1 to 5 , the selected IMFs include considerable fault-related information. As the number increases from 5 to 12, the selected IMFs contain an excessive amount of fault-unrelated information or a high degree of overlap. As the number of selected IMFs increases, the accuracy exhibits the opposite trend. Thus, the first five sensitive IMFs with a large correlation coefficient are considered for subsequent reconstruction in this paper.

To observe the clustering result of the proposed method, the principal component analysis (PCA) is performed on the input and output data of the third hidden layer. A certain confusion occurs among the four conditions in the input data, as shown in Figures 8(a) and 9(a). After the 3 hidden layers of the DBN, as shown in Figures 8(b) and 9(b), most 
TABLE 4: Accuracy comparison for the six methods.

\begin{tabular}{lcc}
\hline Methods & $\begin{array}{c}\text { Accuracy for dataset A (\%) } \\
\text { Training/testing samples }\end{array}$ & $\begin{array}{c}\text { Accuracy for dataset B (\%) } \\
\text { Training/testing samples }\end{array}$ \\
\hline DBN & $72.8 / 71.9$ & $73.4 / 72.4$ \\
EEMD + DBN & $86.8 / 84.7$ & $86.9 / 85.1$ \\
CEEMDAN + DBN (the proposed method) & $93.0 / 92.1$ & $93.6 / 92.5$ \\
CEEMDAN + BP & $55.6 / 54.2$ & $58.2 / 58.0$ \\
$T+$ BP & $40.1 / 39.6$ & $40.6 / 39.8$ \\
$T+F+$ BP & $61.8 / 61.5$ & $63.3 / 62.4$ \\
\hline
\end{tabular}

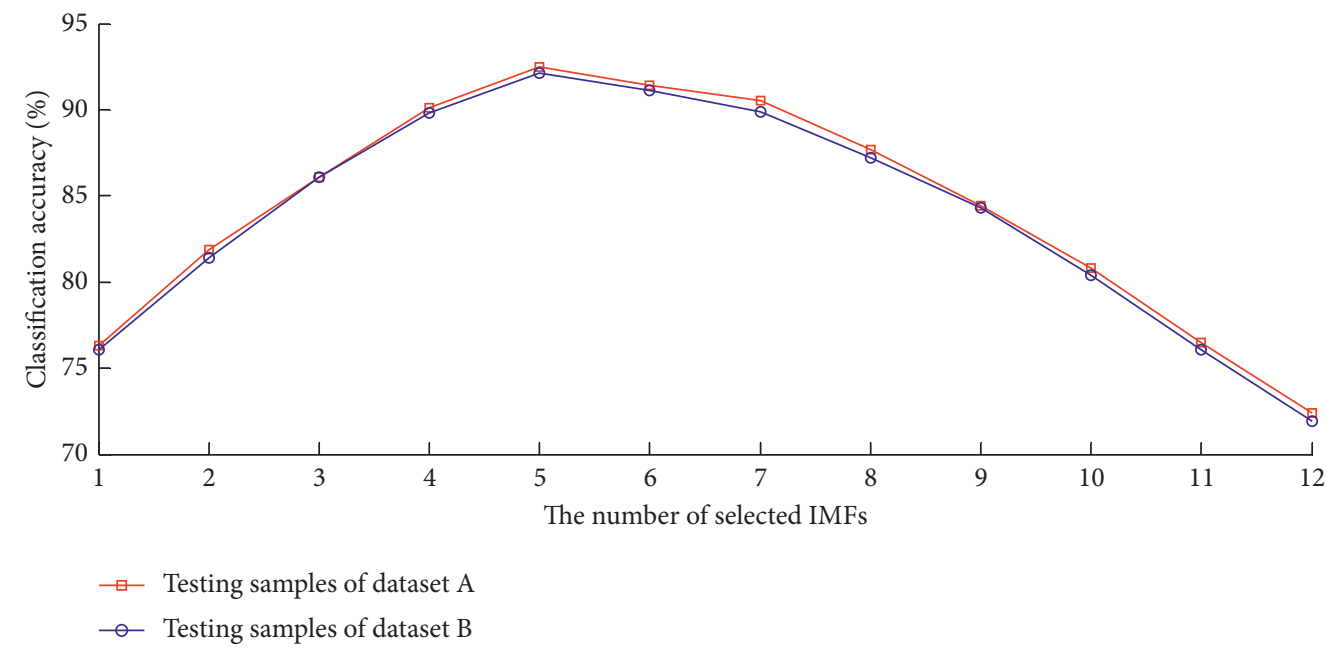

Figure 7: Average classification accuracy under different numbers of selected IMFs.

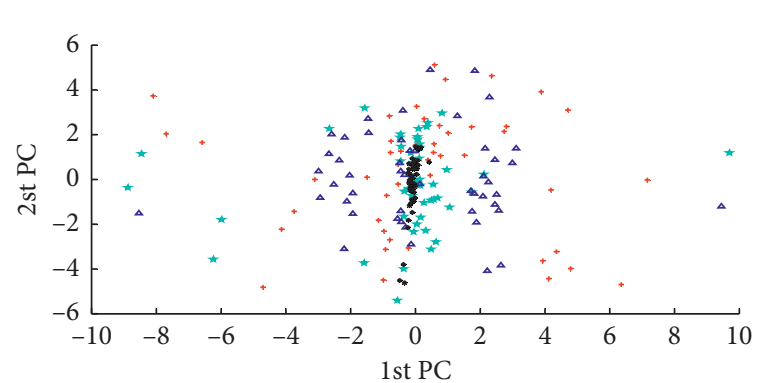

+ No internal leakage

* Small internal leakage

$\triangle$ Medium internal leakage

- Large internal leakage

(a)

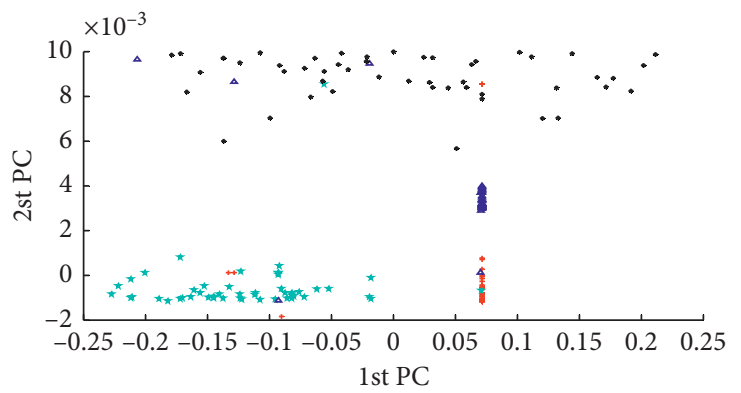

+ No internal leakage $\quad \Delta$ Medium internal leakage

* Small internal leakage • Large internal leakage

(b)

FIgURE 8: 2D-PCA projection of the testing samples of dataset A. (a) Input data. (b) Third hidden layer.

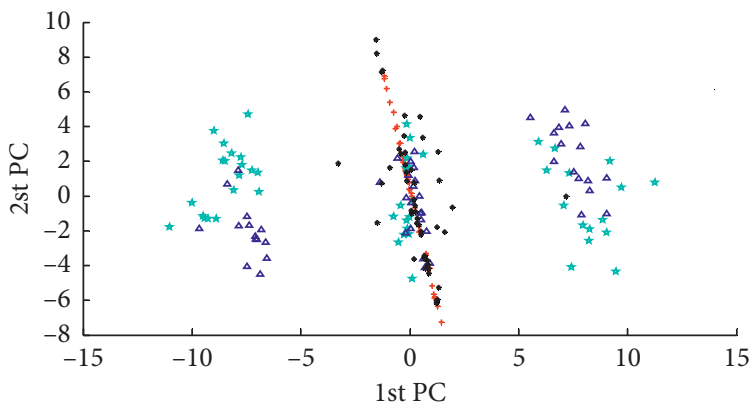

+ No internal leakage $\quad \Delta$ Medium internal leakage

(a)

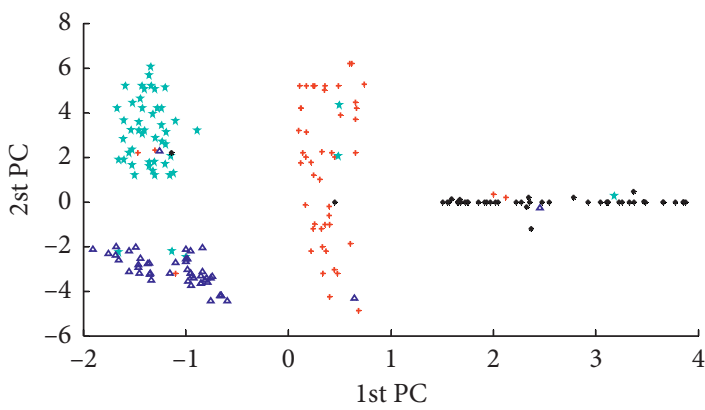

* No internal leakage $\quad \Delta$ Medium internal leakage

* Small internal leakage * Large internal leakage

(b)

Figure 9: 2D-PCA projection of the testing samples of dataset B. (a) Input data. (b) Third hidden layer. 
samples are correctly assigned to the corresponding cluster. The results indicate the effectiveness of the proposed method in mining the fault characteristics and classifying internal leakage conditions.

\section{Conclusions}

This paper proposes a fusion method that combines the optimization DBN with the CEEMDAN to realize the internal leakage diagnosis of hydraulic cylinders under different low loads. The main contributions of the paper are as follows: (1) first, the AE signals are examined to be used for the hydraulic cylinder internal leakage diagnosis. Based on the $\mathrm{AE}$ fault signals, the CEEMDAN-based denoising method and optimal number of representative IMFs for signal reconstruction are investigated. (2) The optimization DBN is obtained using the hybrid PSOSA algorithm instead of subjective human experience. The performance of the proposed method under identical loads is superior to those of the existing state of the art methods. (3) The proposed method can effectively diagnose faults under different low loads, which cannot be easily realized through the existing methods.

\section{Data Availability}

The data used to support the findings of this study are available from the corresponding author upon request.

\section{Conflicts of Interest}

The authors declare no conflicts of interest.

\section{Acknowledgments}

This research was funded by the National Natural Science Foundation of China, grant number (51975425).

\section{References}

[1] P. Ranjan, G. Wrat, M. Bhola, S. K. Mishra, and J. Das, "A novel approach for the energy recovery and position control of a hybrid hydraulic excavator," ISA Transactions, vol. 99, pp. 387-402, 2020.

[2] J. Koivumäki, W.-H. Zhu, and J. Mattila, "Energy-efficient and high-precision control of hydraulic robots," Control Engineering Practice, vol. 85, pp. 176-193, 2019.

[3] J. Zhao, G. Shen, C. Yang, W. Zhu, and J. Yao, "A robust force feed-forward observer for an electro-hydraulic control loading system in flight simulators," ISA Transactions, vol. 89, pp. 198-217, 2019.

[4] L. Li, Y. Huang, J. Tao, C. Liu, and K. Li, "Featured temporal segmentation method and adaBoost-BP detector for internal leakage evaluation of a hydraulic cylinder," Measurement, vol. 130, pp. 279-289, 2018.

[5] Z. Shi, F. Gu, B. Lennox, and A. D. Ball, "The development of an adaptive threshold for model-based fault detection of a nonlinear electro-hydraulic system," Control Engineering Practice, vol. 13, no. 11, pp. 1357-1367, 2005.

[6] L. An and N. Sepehri, "Hydraulic actuator leakage fault detection using extended Kalman filter," International Journal of Fluid Power, vol. 6, no. 1, pp. 41-51, 2005.
[7] A. Y. Goharrizi and N. Sepehri, "A wavelet-based approach to internal seal damage diagnosis in hydraulic actuators," IEEE Transactions on Industrial Electronics, vol. 57, no. 5, pp. 1755-1763, 2009.

[8] A. Y. Goharrizi, N. Sepehri, and Y. Wu, "A wavelet-based approach for diagnosis of internal leakage in hydraulic actuators using on-line measurements," International Journal of Fluid Power, vol. 11, no. 1, pp. 61-69, 2010.

[9] A. Y. Goharrizi and N. Sepehri, "A wavelet-based approach for external leakage detection and isolation from internal leakage in valve-controlled hydraulic actuators," IEEE Transactions on Industrial Electronics, vol. 58, no. 9, pp. 4374-4384, 2010.

[10] X. Zhao, S. Zhang, C. Zhou, Z. Hu, R. Li, and J. Jiang, "Experimental study of hydraulic cylinder leakage and fault feature extraction based on wavelet packet analysis," Computers \& Fluids, vol. 106, pp. 33-40, 2015.

[11] A. Y. Goharrizi and N. Sepehri, "Internal leakage detection in hydraulic actuators using empirical mode decomposition and Hilbert spectrum," IEEE Transactions on Instrumentation and Measurement, vol. 61, no. 2, pp. 368-378, 2011.

[12] Y. LeCun, Y. Bengio, and G. Hinton, “Deep learning," Nature, vol. 521, no. 7553, pp. 436-444, 2015.

[13] F. Jia, Y. Lei, J. Lin, X. Zhou, and N. Lu, "Deep neural networks: a promising tool for fault characteristic mining and intelligent diagnosis of rotating machinery with massive data," Mechanical Systems and Signal Processing, vol. 72-73, pp. 303-315, 2016.

[14] R. Zhao, R. Yan, Z. Chen, K. Mao, P. Wang, and R. X. Gao, "Deep learning and its applications to machine health monitoring," Mechanical Systems and Signal Processing, vol. 115, pp. 213-237, 2019.

[15] Y. Guo, Y. Zeng, L. Fu, and X. Chen, "Modeling and experimental study for online measurement of hydraulic cylinder micro leakage based on convolutional neural network," Sensors, vol. 19, no. 9, 2019.

[16] W. Kaewwaewnoi, A. Prateepasen, and P. Kaewtrakulpong, "Investigation of the relationship between internal fluid leakage through a valve and the acoustic emission generated from the leakage," Measurement, vol. 43, no. 2, pp. 274-282, 2010.

[17] H. Y. Sim, R. Ramli, S. Ahmad, and F. S. Ming, "Detection and estimation of valve leakage losses in reciprocating compressor using acoustic emission technique," Measurement, vol. 152, pp. $1-15,2020$.

[18] B. Ahn, J. Kim, and B. Choi, "Artificial intelligence-based machine learning considering flow and temperature of the pipeline for leak early detection using acoustic emission," Engineering Fracture Mechanics, vol. 210, pp. 381-392, 2019.

[19] X. Lang, Z. Hu, P. Li, Y. Li, J. Cao, and H. Ren, "Pipeline leak aperture recognition based on wavelet packet analysis and a deep belief network with ICR," Wireless Communications and Mobile Computing, vol. 2018, Article ID 6934825, 8 pages, 2018.

[20] G.-Y. Ye, K.-J. Xu, and W.-K. Wu, "Standard deviation based acoustic emission signal analysis for detecting valve internal leakage," Sensors and Actuators A: Physical, vol. 283, pp. 340-347, 2018.

[21] G. Thompson and G. Zolkiewski, "An experimental investigation into the detection of internal leakage of gases through valves by vibration analysis," Proceedings of the Institution of Mechanical Engineers, Part E: Journal of Process Mechanical Engineering, vol. 211, no. 3, pp. 195-207, 1997. 
[22] S. A. Mirhadizadeh, E. P. Moncholi, and D. Mba, "Influence of operational variables in a hydrodynamic bearing on the generation of acoustic emission," Tribology International, vol. 43, no. 9, pp. 1760-1767, 2010.

[23] L. Wang and Y. Shao, "Fault feature extraction of rotating machinery using a reweighted complete ensemble empirical mode decomposition with adaptive noise and demodulation analysis," Mechanical Systems and Signal Processing, vol. 138, Article ID 106545, 2020.

[24] B.-S. Peng, H. Xia, Y.-K. Liu, B. Yang, D. Guo, and S.-M. Zhu, "Research on intelligent fault diagnosis method for nuclear power plant based on correlation analysis and deep belief network," Progress in Nuclear Energy, vol. 108, pp. 419-427, 2018.

[25] Y. Zhang, J. Ji, and B. Ma, "Fault diagnosis of reciprocating compressor using a novel ensemble empirical mode decomposition-convolutional deep belief network," Measurement, vol. 156, Article ID 107619, 2020.

[26] Y. Zou, Y. Zhang, and H. Mao, "Fault diagnosis on the bearing of traction motor in high-speed trains based on deep learning," Alexandria Engineering Journal, vol. 90, Article ID 102085,2020

[27] G. Zhao, X. Liu, B. Zhang, Y. Liu, G. Niu, and C. Hu, “A novel approach for analog circuit fault diagnosis based on deep belief network," Measurement, vol. 121, pp. 170-178, 2018.

[28] J. Gai, J. Shen, Y. Hu, and H. Wang, "An integrated method based on hybrid grey wolf optimizer improved variational mode decomposition and deep neural network for fault diagnosis of rolling bearing," Measurement, vol. 162, Article ID 107901, 2020.

[29] S. Du, J. Lv, and L. Xi, "Degradation process prediction for rotational machinery based on hybrid intelligent model," Robotics and Computer-Integrated Manufacturing, vol. 28, no. 2, pp. 190-207, 2012.

[30] Y. Wang, C. Zhang, and M. Li, "Application of a hybrid algorithm-PSOSA in well test parameter estimation," Petroleum, vol. 4, no. 4, pp. 430-436, 2018.

[31] H. Shao, H. Jiang, X. Zhang, and M. Niu, "Rolling bearing fault diagnosis using an optimization deep belief network," Measurement Science and Technology, vol. 26, no. 11, Article ID 115002, 2015.

[32] M. A. Colominas, G. Schlotthauer, and M. E. Torres, "Improved complete ensemble EMD: a suitable tool for biomedical signal processing," Biomedical Signal Processing and Control, vol. 14, no. 14, pp. 19-29, 2014.

[33] C. He, P. Niu, R. Yang, C. Wang, Z. Li, and H. Li, "Incipient rolling element bearing weak fault feature extraction based on adaptive second-order stochastic resonance incorporated by mode decomposition," Measurement, vol. 145, pp. 687-701, 2019.

[34] G. E. Hinton, "A practical guide to training restricted Boltzmann machines," Neural Networks: Tricks of the Trade, pp. 599-619, Springer, Berlin, Germany, 2012.

[35] P. Tamilselvan and P. Wang, "Failure diagnosis using deep belief learning based health state classification," Reliability Engineering \& System Safety, vol. 115, pp. 124-135, 2013.

[36] L.-l. Li, L. Wang, and L.-h. Liu, "An effective hybrid PSOSA strategy for optimization and its application to parameter estimation," Applied Mathematics and Computation, vol. 179, no. 1, pp. 135-146, 2006.

[37] Y. Qi, C. Li, P. Jiang, C. Jia, Y. Liu, and Q. Zhang, "Research on demodulation of FBGs sensor network based on PSO-SA algorithm," Optik, vol. 164, pp. 647-653, 2018.
[38] K. Thangavel, J. Bagyamani, and R. Rathipriya, "Novel hybrid PSO-SA model for biclustering of expression data," Procedia Engineering, vol. 30, pp. 1048-1055, 2012. 